\title{
Implementation of Networked Control Systems using TCP/IP
}

\author{
Rajesh. R \\ Assistant Professor (Senior Grade) \\ School of Electronics Engineering, \\ VIT University, Vellore, India.
}

\author{
Lakshmanan. M \\ Assistant Professor (Senior Grade) \\ School of Electronics Engineering, \\ VIT University, Vellore, India.
}

\author{
Noor Mohammed. V \\ Assistant Professor (Senior Grade) \\ School of Electronics Engineering, \\ VIT University, Vellore, India.
}

\begin{abstract}
This paper provides a general framework of Networked Control Systems. An Internet based NCS is simulated and TCP/IP communication issue of data streams in Networked Control Systems (NCS) is evaluated. The network is simulated with the ns-2 package, a widespread state-of-the-art open-source network simulator package for studying packet dynamics in networks. The ns-2 release is extended in order to simulate the transmissions of plants and controllers that are modelled by ODEs (solved via a linked package).The results show the overall control and network performances achieved while modelling the individual control and network components. The performance results of different types of data streams in NCS are also presented. Stability issues of NCS are being explored.
\end{abstract}

\section{Keywords}

Networked Control Systems, TCP/IP, NS-2 simulation model.

\section{INTRODUCTION}

Technological advances are delivering devices that have sensing and communication capabilities and that can be ubiquitously embedded in the physical world. Networked control systems are control systems comprised of the system to be controlled and of actuators, sensors, and controllers, the operation of which is coordinated via a communication network. The study of networked control systems (NCSs) brings together the historically separate disciplines of computer networks and control theory. These systems are typically spatially distributed, may operate in an asynchronous manner, but have their operation coordinated to achieve desired overall objectives.

NCS adopts Ethernet as the controlling bus and is well compatible with the Ethernet and Communication network technology. NCS unites the lower control network protocols and establishes wellcompatible interface, thus it is now a hot research area. In fact, NCS should be regarded as a control system based on Internet, and should not be limited to local network. In this sense, NCS can realize such tasks as remote control, remote monitor and remote diagnose, which is hardly realized by traditional network control systems [10]. NCSs are being adopted in many application areas for a number of reasons including their low cost, reduced weight, and power requirements, simple installation and maintenance and higher reliability.

To realize such an Internet based control, the key issue is how to realize an unseemly and safely link between the field control network and the outside network. The policy adopted to solve this problem is through TCP/IP [9] protocol stack to realize the link between the inside and outside networks. There are many mature applications such as EtherNet/IP, HSE Industrial Ethernet, EPA technology, etc, which use Ethernet plus TCP/IP scheme to solve the problem. In these schemes, to inherent TCP/IP as the transmission protocol is undoubtedly a good solution to be compatible with the TCP/IP network of the Internet, but problems arise that control network is quite different from communication networks in that the former has a more strictly requirement for real time and reliability. This paper will simulate an Internet based NCS. It studies the different data stream TCP/IP communication in NCS and the performance parameters.

\section{NETWORKED CONTROL SYSTEMS - AN OVERVIEW}

Traditionally, a networked control system is defined as: a closeloop feedback control system through network is regarded as the networked control system. A networked control system (NCS) consists of numerous physical and computational elements or agents [3], which have physical and informational interactions and dependencies, supported by overlapping network resources. Here, an agent could be a desktop computer; merely a sensor, an actuator, or a control module; or, hierarchically, an ensemble of such devices.

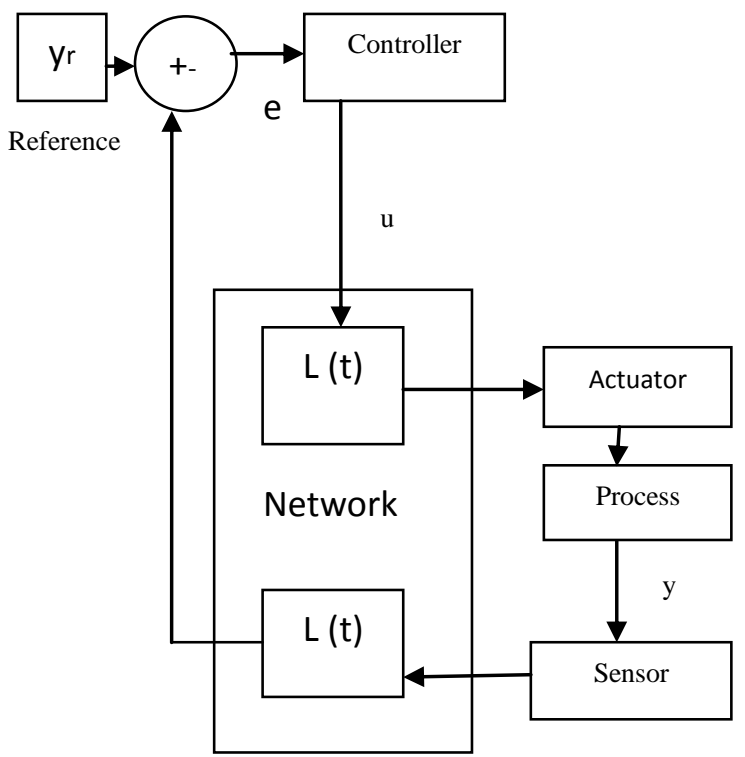

Fig 1 Fully-distributed NCS 
Whenever there is distributed sensing, actuation, or computing of such kind, an NCS involves communication patterns in which both informational and physical control loops are closed through a real-time network. Fig.1 shows a fully-distributed NCS where controller, actuator and sensor are distributed and connected with a network.

The process variable (y) to be controlled is measured and the measurement is transmitted to the controller via the network. The controller compares it with a reference (yr), the desired response, and calculates the control output $(\mathrm{u})$ based on the error signal (e).The controller output is transmitted over the network to the actuator that acts on the process to adjust the controlled variable [4].

\section{NCS-DATA STREAM TYPES}

Network delay is produced in the process of data transmission. Whether the delay will cause bad effects to all the data is uncertain, which is closely related to the type of data transmitted and the real-time requirement of these data. In NCS, the data transmitted are classified to three types: periodic data, sudden data and non-real time data [1].

\subsection{Periodic data}

The periodic data refers to I/O data of sensors and controllers and some system status monitor data. The periodic data have a strict limit to time. A small delay is not allowed in the periodic data transmission, in some cases, even microsecond time delay is not allowed. Besides, for the periodic data, only the updated data are useful, if within the demand time cycle the data do not arrive or data error occurs, even the next cycle data have been generated, the data should be discarded eventually. So, in most cases, the periodic data do not require a retransmission.

\subsection{Sudden data}

This type of data includes alarm and urgent operation signals. The sudden data have the most real time requirement, which have more priority over the periodic data. The sudden data require an accurate data transmission. Generally, the length of the sudden data is short and its volume is relatively low, which utilize little bandwidth of the network.

\subsection{Non-real time data}

The non-real-time data are composed of programming and configuration data, which have low real time requirement and permit certain time delay in transmission. The length of non-real time data is long and varies frequently. The volume of non-real time data is great, in most cases, they appear as a mini file or small file, which has low bandwidth occupation in transmission. But this type of data is useful and is not allowed to lose. So fault control and retransmission policy are required to guarantee the integrity and accuracy of the transmitted data. In the above three types of data, the periodic data and sudden data are real time data. They have different real time requirements. The former should meet the time limit requirement and is sensitive to time delay whose uncertainty would affect the stability of the closed-loop control and the performance of the control quality. The sudden data require to be reacted as soon as possible and the transmission delay as short as possible while the delay uncertainty is not considered. The sudden data have more reliable requirements than the periodic data.

\section{NCS STABILITY ANALYSIS}

The insertion of communication networks into feedback control loops makes the analysis and design of an NCS complex. Conventional control theories with many ideal assumptions (such as equal-distance sampling, synchronized control, and nondelayed sensing and actuation) must be re-evaluated before they can be applied to NCSs.

Specially, the following fundamental issues are of concern, (1) time-varying transmission period, (2) network schedulability, (3) network-induced delay (or latency), and (4) packet loss.

The transmissions of information on an NCS can be periodic or a periodic, depending on the Medium Access Control (MAC) protocol of the control network. So, the first issue is, NCSs with time-varying transmission period. This results in a time-varying system model making stability analyses non-routine.Network scheduling is the primary issue in NCS design when a set of agents are connected over the available network resources. With no coordination, concurrent transmissions will occur and someone has to back to avoid collisions or bandwidth violations. This results in transmissions of some real-time data being delayed or even missing their deadlines. Good control scheduling algorithms will try to minimize such system performance loss.

Another issue is the network-induced delay [8] that occurs while exchanging data among agents connected to the shared medium. This delay, either constant or time-varying, can degrade the performance of control systems designed without considering it and can even destabilize the system. One more issue is that networks can be viewed as a web of unreliable transmission paths. Some information packets not only suffer transmission delay, but even worse, can be lost during transmission. Thus, how such packet dropouts affect the performance of an NCS is an issue that must be considered. Finally, plant outputs may be transmitted using multiple network packets (so-called multiple-packet transmission), due to the bandwidth and packet-size constraints of the network. Because of the arbitration of the network medium with other nodes on the network, chances are, all/part/none of the packets could arrive at the time of control calculation. NCS stability theorem [5], [6] is based on Multiple Lyapunov Function approach, which aims to guarantee the decrease of a Lyapunov function at every transmission event.

\subsection{Control and scheduling co-design}

Consider the case of a set of agents that are connected to a network. Each agent has a required transmission rate of their data in order for the interactions among agents to be stable. However, when the transmission path is shared with other agents, transmission scheduling among the plants has to be considered. Concepts of network scheduling in NCSs may be extended from those for CPU scheduling [2]. Both cases involve allocating a shared resource to a set of concurrent tasks; both involve frequent invocations of concurrent tasks, and both tasks have real-time constraints and have deadlines to be met. However, in the case of network scheduling in NCSs, the shared resource becomes the network instead of the CPU processor, and the execution of a realtime task has been replaced by the transmission of a data packet. 


\section{NCS SIMULATION BASED ON NS-2}

\subsection{Topology of the network \& its parameters} The network is simulated with the ns-2 package, and the ns-2 release is extended to simulate the transmissions of plants and controllers. Specifically, we can create a new agent to represent a plant. Such an agent samples its output at a specified interval, writes the sample output into a packet, and sends it to the controller. The ns- 2 simulator will then follow the packet through the network as it is transmitted over links, queued at router buffers, dequeued, an finally reaches the controller. The controller immediately generates a control $u$ and writes $u$ in a packet that is delivered through the network to the plant. Fig 2 shows the graphical interface of the simulator as it is running an experiment with 3 plants linked to a controller through an intermediate router.

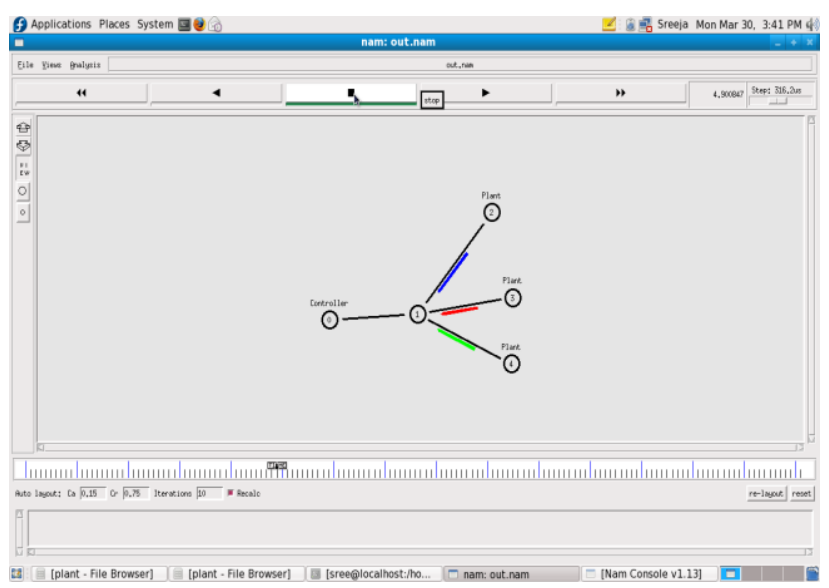

Fig 2 Topology of NCS in ns-2

The plants (nodes 2 to 4 ) are connected to a Fast Ethernet switch (node 1). The plant-switch links have a $120 \mu$ s latency, which includes propagation delay as well as per-frame processing at the end-points. Plant 2 is a distinguished plant, whose output will be traced over time. The other plants are added to simulate a scenario where plant 2 is in contention with other units for network resources. Plant 2 samples its output every $100 \mathrm{~ms}$. Output samples are represented by double-precision floating point numbers. The switch is connected to the controller through a T1 line with a $1 \mathrm{~ms}$ delay. In terms of packet dynamics, the fundamental metrics are: (1) delays (one-way from plant to controller); (2) drop rate (the fraction of sample packets dropped), and (3) inter-arrival gaps (time between reception of successive control packets at the plants).

\subsection{Plant and controller dynamics}

The distinguished plant (Plant 2) has a scalar output defined by $\mathrm{x}_{-}(\mathrm{t})=\mathrm{Ax}(\mathrm{t})+\mathrm{u}(\mathrm{t}) ; \mathrm{y}(\mathrm{t})=\mathrm{x}(\mathrm{t})$; where $\mathrm{u}(\mathrm{t})$ is the plant input. The initial plant state is -1 . If the output is sampled at time $t$, the (subsequently computed and sent) control signal comes back to the plant at time $t+\operatorname{hrtt}(t)$, where $\operatorname{hrtt}(t)$ is the round-trip time for feedback to arrive from the plant output measurement taken at time $t$. The controller predicts the plant state forward by $3.024 \mathrm{~ms}$ (the nominal round-trip time in an unloaded network) and then uses a simple proportional scheme $\mathrm{u}=-0.8 \mathrm{y}$ to achieve the reference $y=0$. Differential equations for the plant and the controller are solved numerically with the Ode utility.

\section{SIMULATION RESULTS \& ANALYSIS}

Table 1 Simulated communication tasks

\begin{tabular}{|c|c|c|c|}
\hline Data Type & $\begin{array}{c}\text { Real Time } \\
\text { Data }\end{array}$ & Sudden Data & $\begin{array}{c}\text { Non Real } \\
\text { Time Data }\end{array}$ \\
\hline Protocol & UDP & UDP + TCP & TCP \\
\hline $\begin{array}{c}\text { Packet Size } \\
\text { (bytes/packet) }\end{array}$ & 1024 & 100 & $>1024$ \\
\hline
\end{tabular}

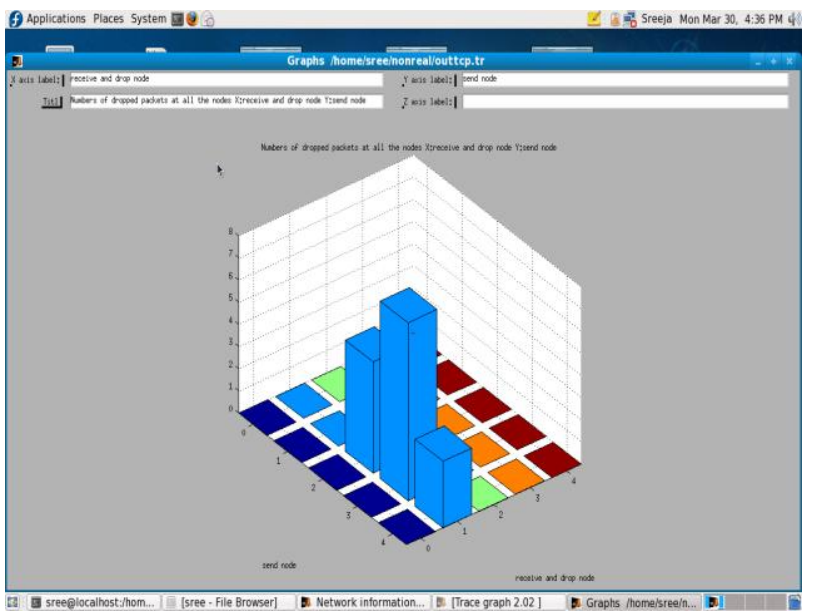

Fig 3 Drop out IP rate of non-real time data.

Fig 3 shows the Maximum drop out IP rate during the transmission of non real time data over time. The simulation time is set at 360 seconds.

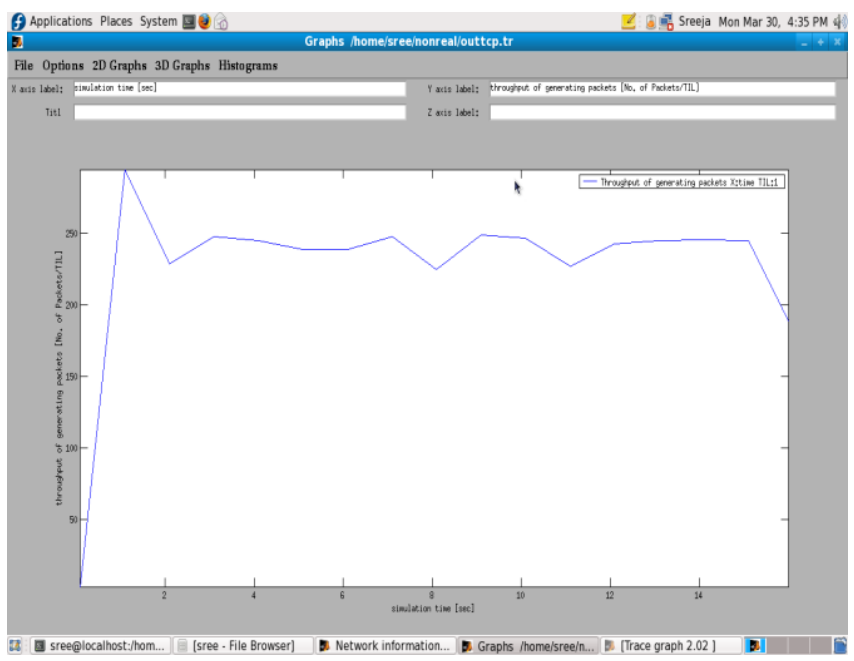

Fig 4 Throughput of non real time data transmission 
Fig 4 shows the simulated graph of throughput Vs time for the non real time data transmission. TCP/IP protocol is applied for the transmission of the non real time data. The simulation time is set at 360 seconds.

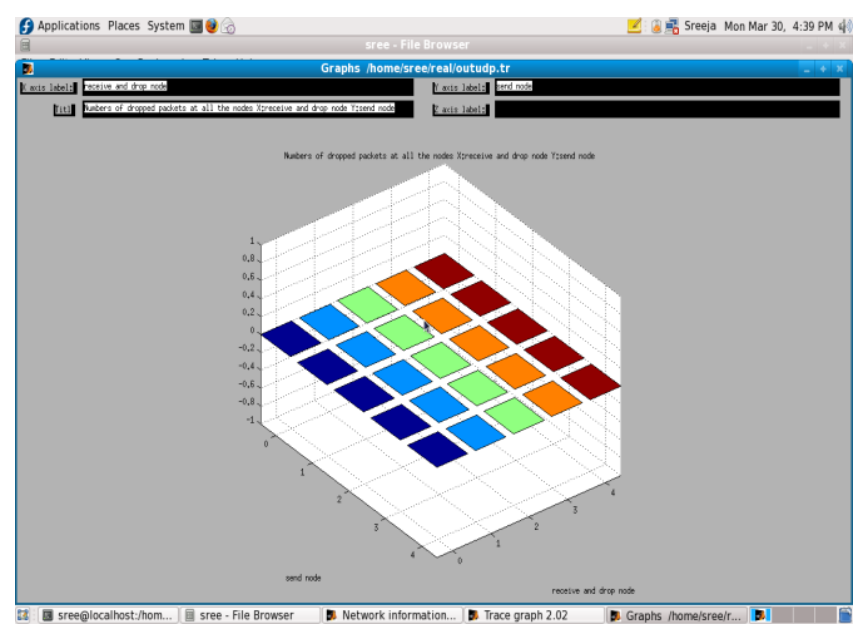

Fig 5 Drop out IP rate of real time data

Fig 5 shows the real time data transmitted in UDP.Here there are no packet drop outs. The UDP protocol uses a non link and no reliable mechanism. Thus the real time performance superior to the TCP. The simulation time is set at 360 seconds.

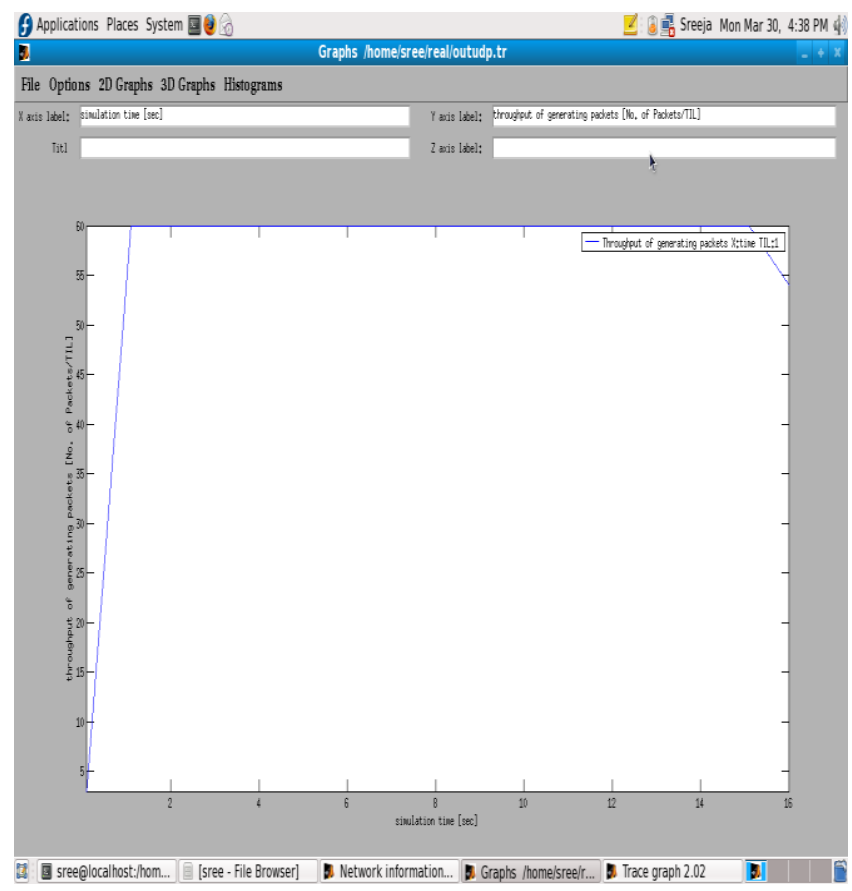

Fig 6 Throughput of periodic data transmission

Fig 6 shows the simulated graph of throughput vs time for the real time data transmission using UDP /IP protocol. The simulation time is set at 360 seconds.

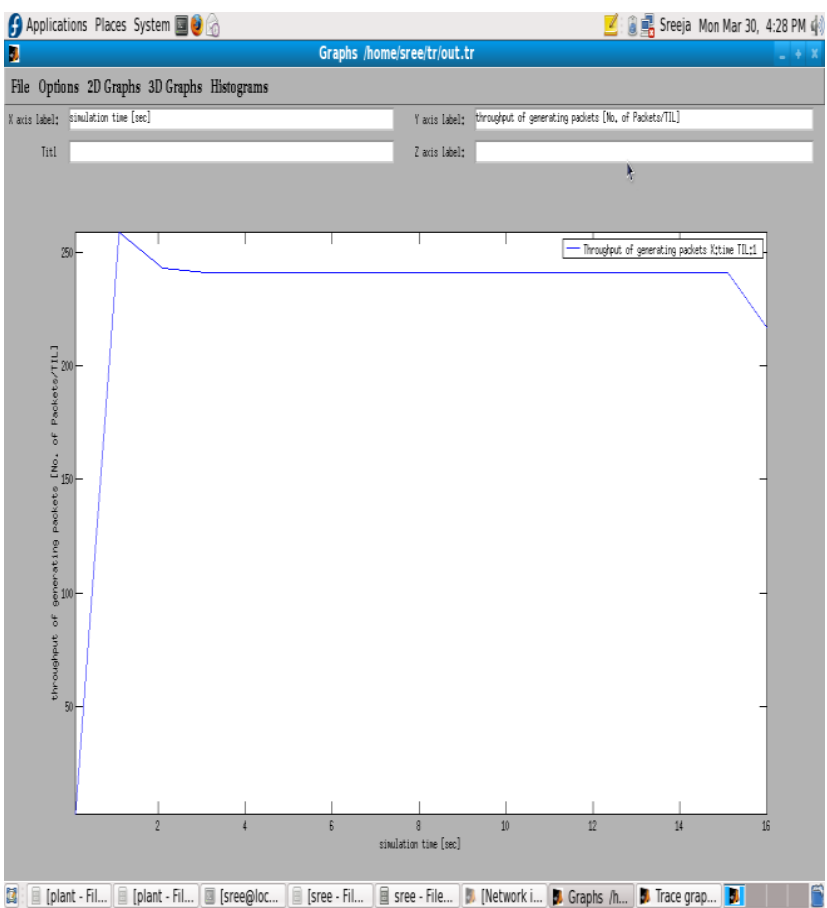

Fig 7 Throughput of Sudden data transmission

The sudden data are transmitted through TCP and UDP protocols.It can guarantee both real time and reliability. Fig 7 shows throughput vs time of sudden data transmission.The simulation time is set at 360 seconds.

\section{CONCLUSION}

This paper has evaluated he performance issues of TCP/IP communication of the data streams of NCS. Simulation results show that real time and reliability can be guaranteed by applying TCP or UDP to different data. But the real time of TCP protocol should be improved for better performance and the reliability of the UDP protocol should be strengthened. Researches must be done to improve the TCP/IP performance to match the NCS communication requirements.

\section{REFERENCES}

[1] An Wei, Yuqiang Chen and Jinhua Wu, 2006, "Simulation study of TCP/IP communication based on networked control Systems", Proceedings of the $6^{\text {th }}$ world congress on intelligent control and application, June21-23, Dalian, China.

[2] Michael S. Branicky, Vincenzo Liberatore and Stephen M. Philip.,."Ncs Co-Simulation for Co-Design”.

[3] Vincenzo Liberatore, 2002,"Network controls Systems".

[4] Michael Pohjola, 2006, "PID Contoller Design in NCS", Master's thesis.

[5] Zhang. W, Branicky .M. S, Philips S M., 2001, "Stability of networked control systems," IEEE Control System Managemnt., pp.84 88. 
[6] G. C. Walsh, H. Ye, and L Bushnell, 1999, "Stability analysis of networked control systems," Proc Amer, Control Conf, SanDiego, CA, pp.2876-2880.

[7] G. C. Walsh, O. Beldiman, and L Bushnell, 1999 "Error encoding algorithms for networked control systems," Proc of the 38th Conf On Decision \& Control Phoenix, Arizona USA, pp.4933-4938.
[8] Yang Liman, Li Yunhua, and Yuan Haibing, 2004,"Analysis of time delay in networked control systems and study of data transmission technology," Control and Decision, pp.361-382.

[9] M. Mellia, I. Stoica, and H, Zhang, "TCP model for short lived Flows", IEEE Communication Letters, vol.6, No:2, pp 85-87.

[10] Yang Xianhui. 2003, "Industrial Data Communication and Control Network", Tsinghua University Press. 